\title{
Contribuições da análise da atividade do trabalho no processo de retorno do trabalhador afastado por LER/DORT*
}

\section{Contributions of the analysis of labor activity in the employee's return process after leave of absence due to RSI/WMSDs}

\author{
Elaine Cristina Silva ${ }^{1}$, João Alberto Camarotto ${ }^{2}$
}

http://dx.doi.org/10.11606/issn.2238-6149.v27i2p131-137

Silva EC, Camarotto JA. Contribuições da análise da atividade do trabalho no processo de retorno do trabalhador afastado por LER/DORT. Rev Ter Ocup Univ São Paulo. 2016 maio-ago.;27(2):131-7.

RESUMO: O aumento do número de trabalhadores afastados por LER/DORT tem constrangido a sociedade brasileira a lidar com esse problema, visto que a importância de suas manifestações pode ser considerada um grave problema de saúde pública. Os trabalhadores afastados têm encontrado várias dificuldades ao retornar ao trabalho, uma vez que, além das limitações funcionais, também enfrentam obstáculos no trabalho. Além disso, a reabilitação profissional do INSS, não garante as melhorias necessárias para um efetivo retorno às atividades laborais. Frente a esta realidade, o objetivo deste artigo é proporcionar o entendimento de como a análise da atividade de trabalho contribui para o processo de retorno de trabalhadores afastados por LER/DORT. O modelo proposto no fluxograma para a análise do processo de retorno ao trabalho, após afastamento por LER/DORT, é explicado e associado aos pressupostos da Ergonomia da Atividade. A análise da atividade de trabalho permite a compreensão dos aspectos intrínsecos das ações dos trabalhadores e os respectivos constrangimentos, o que permite aproximar a necessidade da empresa com as potencialidades dos trabalhadores.

DESCRITORES: Retorno ao trabalho; Transtornos traumáticos cumulativos; Engenharia humana; Trabalhadores/psicologia.
Silva EC, Camarotto JA. Contributions of the analysis of labor activity in the employee's return process after leave of absence due to RSI/WMSDs. Rev Ter Ocup Univ São Paulo. 2016 May-Aug.;27(2):131-7.

ABSTRACT: The increase in the number of employees on leave for RSI/WMSDs has embarrassed the Brazilian society when dealing with this problem, since the importance of its manifestations can be considered a serious public health problem. Employees on leave have been finding several difficulties in returning to work, since, in addition to functional limitations, they also face obstacles at work. Furthermore, the vocational rehabilitation of INSS does not ensure necessary improvements for an effective return to labor activities. Before this reality, this article aims to provide an understanding of how the analysis of labor activity contributes to the return process of employees on leave for RSI/WMSDs. The model proposed in the flowchart for the analysis of the work return process, after leave for RSI/WMSDs, is explained and associated with the hypotheses of the Activity Ergonomics. The analysis of labor activity allows the understanding of intrinsic aspects of the actions of the employees and their respective constraints, allowing to approach the need of the company with the potential of employees.

KEYWORDS: Return to work; Cumulative trauma disorders; Human engineering; Workers/psychology.

*Este artigo é parte da pesquisa de doutorado desenvolvido no Programa de Pós-Graduação em Engenharia de Produção da Universidade Federal de São Carlos (UFSCar) e financiado pela CAPES. Linha de pesquisa: Trabalho, Tecnologia e Organização (TTO).

1. Terapeuta Ocupacional, mestre e doutoranda do Programa de Pós-graduação em Engenharia de Produção da Universidade Federal de São Carlos (UFSCar), São Carlos, SP, Brasil. E-mail: elainegpec@gmail.com

2. Professor Titular do Departamento de Engenharia de Produção, Universidade Federal de São Carlos (UFSCar), São Carlos, SP, Brasil. E-mail: camarotto@dep.ufscar.br

Endereço para correspondência: DEP/UFSCar - Rod. Washington Luis, Km 235. Caixa Postal: 676. CEP: 13 565-905 - São Carlos, SP. E-mail: elainegpe@gmail.com 


\section{INTRODUÇÃO}

$\mathrm{U}$ $\mathrm{m}$ dos destaques para o perfil epidemiológico dos adoecimentos relacionados ao trabalho são as Lesões por Esforços Repetitivos/ Distúrbios Osteomusculares Relacionados ao Trabalho (LER/ DORT), que em conjunto com os transtornos psíquicos e acidentes de trabalho, constituem as principais causas dos afastamentos, causas estas atribuídas às novas exigências do trabalho1. Percebe-se que cada vez mais as tarefas tem requerido iniciativa, comunicação e trocas entre os trabalhadores, ou seja, maneiras variáveis no processo de ação e reconhecimento de competências ${ }^{2}$.

O aumento considerável do número de trabalhadores afastados por LER/DORT, tem constrangido a sociedade brasileira a lidar com esse problema, visto que a importância de suas manifestações é considerada um problema de saúde pública.

$\mathrm{O}$ número de acidentes com Comunicação de Acidente de Trabalho (CAT) no Brasil relacionadas à doença foi de 15.226 casos em 2013, sendo que os códigos do CID10 (Classificação Estatística Internacional de Doenças e Problemas Relacionados com a Saúde) mais incidentes foram de dorsalgia, lesões do ombro, sinovite e tenossinovite ${ }^{3}$.

$\mathrm{Na}$ primeira fase do processo de adoecimento osteomuscular, a invisibilidade da origem da dor gera dúvidas quanto ao nexo da doença com o trabalho. Este fato, associado à preocupação com os aspectos psicológicos e biomecânicos, deixa de lado o aspecto psicossocial, o que contribui para acentuar o preconceito em torno do indivíduo afastado por LER/DORT.

A visão biomédica, que salienta a necessidade de evidência física da lesão para a caracterização das LER/ DORT, torna difícil o seu reconhecimento na fase inicial, quando a manifestação ocorre pela dor ou desconforto ${ }^{4}$.

Diante do diagnóstico de LER/DORT, o médico responsável verifica o estágio da doença para decidir sobre o possível afastamento do trabalhador. Constatada a necessidade de afastamento e reabilitação, o trabalhador é encaminhado para um perito médico do Instituto Nacional do Seguro Social (INSS), que o avalia e o encaminha, de acordo com suas condições/capacidades, seguindo três situações distintas: a) $\mathrm{O}$ trabalhador com incapacidade permanente é encaminhado para a aposentadoria; b) o trabalhador para o qual não se verifica alguma incapacidade retorna diretamente para a empresa; e c) no caso em que o trabalhador apresenta uma incapacidade temporária, este é encaminhado para o Programa de Reabilitação Profissional do INSS. Este processo está previsto na Constituição para que os trabalhadores possam participar do mercado de trabalho e do contexto em que vivem. Para isso, o Programa de Reabilitação Profissional deve proporcionar os meios de readaptação profissional e social .

O processo de inclusão da pessoa que retorna ao trabalho é realizado por meio do Programa de Reabilitação Profissional do INSS, porém, não se identificam neste, quesitos que garantam a completa reabilitação, com efetiva inclusão ou retorno do trabalhador ao ambiente social de trabalho ${ }^{6}$.

Os trabalhadores, acometidos por LER/DORT, encontram dificuldades ao retornar ao trabalho, visto que, além das limitações funcionais, também enfrentam obstáculos junto ao grupo de trabalho e/ou chefia da empresa, e, muitas vezes, a não adequação ao novo posto de trabalho. Neste processo, o Programa de Reabilitação Profissional do INSS não garante as melhorias necessárias para o retorno ao trabalho ${ }^{7}$.

Considerando que a Ergonomia da Atividade (EA) tem como objetivo entender a situação real de trabalho para transformá-la com efetividade, este artigo busca entender a análise da atividade de trabalho, definida pela EA, como parte da Análise Ergonômica do Trabalho - AET $^{8}$, e de que modo ela contribui para o processo de retorno de trabalhadores afastados por LER/DORT.

Propõe-se, por meio da análise da atividade, compreender o trabalho e reconhecimento da competência necessária para determinado local de trabalho, através da intermediação entre empresa, trabalhador e Programa de Reabilitação Profissional do INSS. AEA possui conceitos que são fundamentais e orientam a compreensão das situações de trabalho, a fim de identificar os determinantes e realizar as possíveis transformações ${ }^{8}$ que tenham um impacto positivo sobre a saúde e produtividade.

O itinerário proposto, também tem por objetivo operacionalizar a inclusão destacada por Lancman et al. ${ }^{9}$, que aborda o retorno ao trabalho e os aspectos necessários para a sua efetividade.

Neste processo, são estudados os postos que os trabalhadores ocupavam por ocasião de respectivos afastamentos e os postos de trabalho para os quais os retornam, ou seja, atividades que foram selecionados pela empresa em comum acordo com o Programa de Reabilitação Profissional do INSS e com o trabalhador; e adaptados para receber os trabalhadores que retornam após afastamento por LER/DORT.

O êxito no retorno e permanência no trabalho está relacionado com o envolvimento de diversos órgãos, campos, atores sociais e profissionais, que devem direcionar suas atuações em torno de um eixo comum e predefinido coletivamente. Caso contrário, os trabalhadores são 
direcionados para postos de trabalho incompatíveis com suas capacidades laborais, formações e qualificações ou são deixados à margem do processo produtivo?

Este estudo faz parte da pesquisa de doutorado da autora no Programa de Pós-graduação em Engenharia de Produção da UFSCar, na linha de pesquisa: Trabalho, Tecnologia e Organização.

\section{O processo de retorno ao trabalho no contexto brasileiro}

De acordo com o Artigo 136, do Decreto 3.048/99 da Previdência Social, a reabilitação profissional é um serviço da Previdência Social, prestado pelo INSS, com o objetivo de proporcionar os meios de reeducação ou readaptação profissional para o retorno ao trabalho dos trabalhadores incapacitados (segurados) por doença ou acidente.

O segurado encaminhado ao Programa de Reabilitação Profissional, após a avaliação médico-pericial, deve, independente da idade e sob pena de suspensão do benefício, submeter-se ao programa, que é prescrito e custeado pela Previdência Social, dispensando tempo mínimo de contribuição para que o segurado tenha direito.

No entanto, a Reabilitação Profissional oferecida pelo INSS enfrenta dificuldades decorrentes da grande demanda, frente ao corpo reduzido de profissionais especializados, além da dificuldade financeira nos programas de reabilitação ${ }^{4}$.

Quanto maior o tempo de afastamento, menor a probabilidade de retorno e permanência no trabalho ${ }^{10}$. De acordo com Meijer et al. ${ }^{11}$, quanto mais rápido ocorre o retorno da licença médica, mais fácil é a adequação ao novo posto de trabalho, após o retorno.

Quando os trabalhadores retornam ao trabalho frequentemente são remanejados para funções incompatíveis com o seu quadro clínico-funcional; com sua formação e qualificação profissional; ou são deixados à margem do processo produtivo, "encostados", o que reforça as experiências de fracasso ${ }^{12}$.

Segundo Saldanha et al. ${ }^{13}$, a demanda por reabilitação profissional é grande e contemplada por Políticas intersetoriais, cooperação técnica e pactuação de metas entre os envolvidos no processo de reinserção no trabalho.

Não são identificadas, ainda, por parte do Ministério da Previdência Social, propostas que incluam aspectos importantes para a efetiva reabilitação profissional para o retorno do trabalhador ao ambiente social de trabalho, ou seja, com "a inserção do trabalhador em um trabalho que permita sua integração social plena".

Para Lancman et al. ${ }^{9}$, o retorno ao trabalho deve acontecer para a mesma função de origem ou em função compatível com a condição de saúde e capacidade laborativa do indivíduo. Sendo que, o sucesso ou o fracasso desse processo depende de vários aspectos, como: organização do trabalho, capacidade profissional, relações interpessoais e capacidade laborativa.

Quando por alguma incapacidade provocada pela atividade laborativa, em particular LER/DORT, as pessoas são excluídas do trabalho temporariamente, podem ficar privadas de suas prerrogativas de integração ao trabalho.

Como é previsto na legislação previdenciária, após o afastamento e retorno do trabalhador que foi acometido, é realizada a visita da equipe do programa de reabilitação do INSS. Porém, as avaliações são pontuais e restritas aos casos de resistência sistemática por conta do retorno ao trabalho. Associada a essa situação, se identifica a fragilidade da equipe em negociar novos postos de trabalho com as empresas, visto que há desconhecimento dos riscos que podem existir no ambiente, no processo e nas relações de trabalho, fato que resulta em baixa fixação dos trabalhadores nas novas atividades. Essa dificuldade de articulação com as empresas, muitas vezes, leva a demissão do trabalhador ou ao retorno deste para a perícia médica, o que torna o processo de reabilitação profissional ineficaz ${ }^{14}$.

A falta do trabalho conjunto entre empresa e órgão público penaliza o trabalhador, este fato identifica a precariedade no tratamento dado pelo poder público às questões relativas à saúde e trabalho, na medida em que trata separadamente estes aspectos que são indissociáveis ${ }^{14}$.

O retorno ao trabalho mostra-se como um desafio, frente às condições que norteiam os trabalhadores afastados por LER/DORT, visto que existem barreiras e facilitadores na constituição desse processo.

\section{Pressuposto Metodológico e Conceitual}

A base teórica metodológica que orienta o trabalho é a Ergonomia da Atividade, que tem por objetivo o estudo da atividade real de trabalho, envolvendo as representações sobre o trabalho daqueles que executam e daqueles que planejam as atividades e como método de análise os fundamentos da $\mathrm{AET}^{8,15}$.

Segundo a $\mathrm{AET}^{8}$, a atividade de trabalho não se restringe ao conjunto de regras previamente conhecidas, mas às regulações, no qual se considera as variabilidades (de produção e dos sujeitos envolvidos no trabalho), da carga de trabalho (física, cognitiva e psíquica) e os modos operatórios (diferentes maneiras de fazer o trabalho, ou seja, a pessoa adota formas diferentes de alcançar os objetivos e os meios que ela opera, de forma a adequar a carga de trabalho ao seu modo de trabalhar, utilizando sua competência e garantindo sua saúde). 
Assim, a atividade é o que o trabalhador efetivamente faz para realizar a tarefa, ou seja, fazer o que é prescrito ou do que deve ser realizado segundo o planejamento da empresa. Portanto, envolve todas as ações do trabalhador para realizar sua parcela do trabalho da produção. Envolve a interação do trabalhador com os artefatos, visando cumprir as tarefas prescritas pela empresa. "A atividade de trabalho se dá em condições reais e produz resultados efetivos"

Segundo os pressupostos da EA, a atividade é compreendida pela observação sistemática, de forma participativa, das situações de trabalho, procurando-se entender como o trabalho é efetivamente realizado ${ }^{8,15}$.

Esta metodologia observa o comportamento humano, analisando de maneira detalhada cada um dos deslocamentos, comunicações, posturas, direção do olhar, condutas, modos operatórios, procedimentos cognitivos, ou seja, o que relaciona o trabalhador à atividade.

\section{MÉTODOS E TÉCNICAS}

Como o objetivo deste artigo é proporcionar o entendimento de como a análise da atividade de trabalho contribui para o processo de retorno de trabalhadores afastados por LER/DORT, tem-se como proposta o estudo de postos responsáveis pelo afastamento e postos escolhidos para a reinserção.

A estrutura proposta para a análise do processo de retorno ao trabalho, após afastamento por LER/DORT, sintetizado no fluxograma ${ }^{17}$ da Figura 1, é baseada no modelo de inclusão da pessoa com deficiência de Simonelli ${ }^{18}$. Isso contribuiu para a compreensão da função dos atores sociais envolvidos no processo e propõe, através do método da AET e da aplicação da CIF, identificar os requisitos técnicos exigidos pela atividade, as barreiras e facilitadores, além das potencialidades dos trabalhadores portadores de deficiência. A estrutura utilizada neste artigo tem como base para a construção do fluxograma de inclusão da pessoa afastada por LER/DORT, o modelo de Simonellii ${ }^{18}$, aplicado à realidade do adoecimento no trabalho e nas percepções e perspectivas dos diferentes atores sociais (analistas do PRP INSS, trabalhadores, empresa), envolvidos no processo a partir de entrevistas, aplicação da análise da atividade de trabalho e avaliação a partir da CIF.

O fluxograma inicia pelo diagnóstico do perito médico do INSS e afastamento do trabalho, seguido por um período de tratamento/reabilitação/readaptação. Em seguida, o trabalhador é encaminhado pelo médico perito, diretamente para o retorno ao trabalho, para aposentadoria por invalidez, ou ainda, para o Programa de Reabilitação Profissional do INSS, caso tenha adquirido uma incapacidade temporária. A partir do Programa de Reabilitação Profissional do INSS, retorna ao trabalho.

O fluxograma apresenta duas representações do mundo do trabalho: de um lado a análise das potencialidades e habilidades do operador que está retornando ao trabalho, após um afastamento por LER/DORT, e de outro, as ações da empresa efetivadas para garantir a reinserção.

A análise das potencialidades do trabalhador evidencia sua capacidade (física e mental) e limitações para o trabalho. A sistematização da capacidade, a partir das potencialidades, facilitadores, experiências e conhecimentos, revelam as tarefas potenciais a serem realizadas pelo trabalhador que retorna ao trabalho.

A parte em destaque no fluxograma (Figura 1), mostra as ações da empresa com suas demandas, seus requisitos técnicos e conhecimentos da tarefa, que orienta a execução do trabalho. Além das características organizacionais e funcionais, que são essenciais na adequação do local e do ambiente laborativo para a reinserção ${ }^{17}$. O destaque sobre as ações da empresa é o objeto deste estudo.

\section{DISCUSSÃO}

A Análise Ergonômica do Trabalho (AET), leva em consideração os trabalhadores, individual e coletivamente, na construção de saúde, competências e, ao mesmo tempo, a relação entre as condições, a atividade e os resultados, representada pelo modelo da função integradora da atividade de trabalho ${ }^{8}$. Além disso, a AET tem por alicerce o ponto de vista da atividade de trabalho, realizada por meio da participação efetiva do trabalhador, para compreender as dimensões física, cognitiva e organizacional da atividade ${ }^{8}$. A compreensão da atividade de trabalho tem por objetivo final transformar o trabalho para torná-lo mais adequado às características psicofisiológicas dos trabalhadores e melhorar a eficácia do sistema de produção ${ }^{16}$.

A adequação do local, do ambiente de trabalho e a confrontação com as competências do trabalhador, facilitam a análise e adaptação da atividade de trabalho às suas capacidades e potencialidades. A partir dos pressupostos metodológicos da EA, com a construção do método de análise baseado nos fundamentos da AET, a adaptação da atividade de trabalho ao operador, posteriormente, também irá necessitar de validação, entre os atores sociais desse processo: trabalhador (ator principal, reinserido no posto de trabalho), empresa (ator responsável pela escolha e adaptação dos postos para a reinserção do trabalhador) e Programa de Reabilitação Profissional do INSS (ator que faz o intermédio entre trabalhador e empresa e que deve fazer parte da escolha do posto de reinserção). 


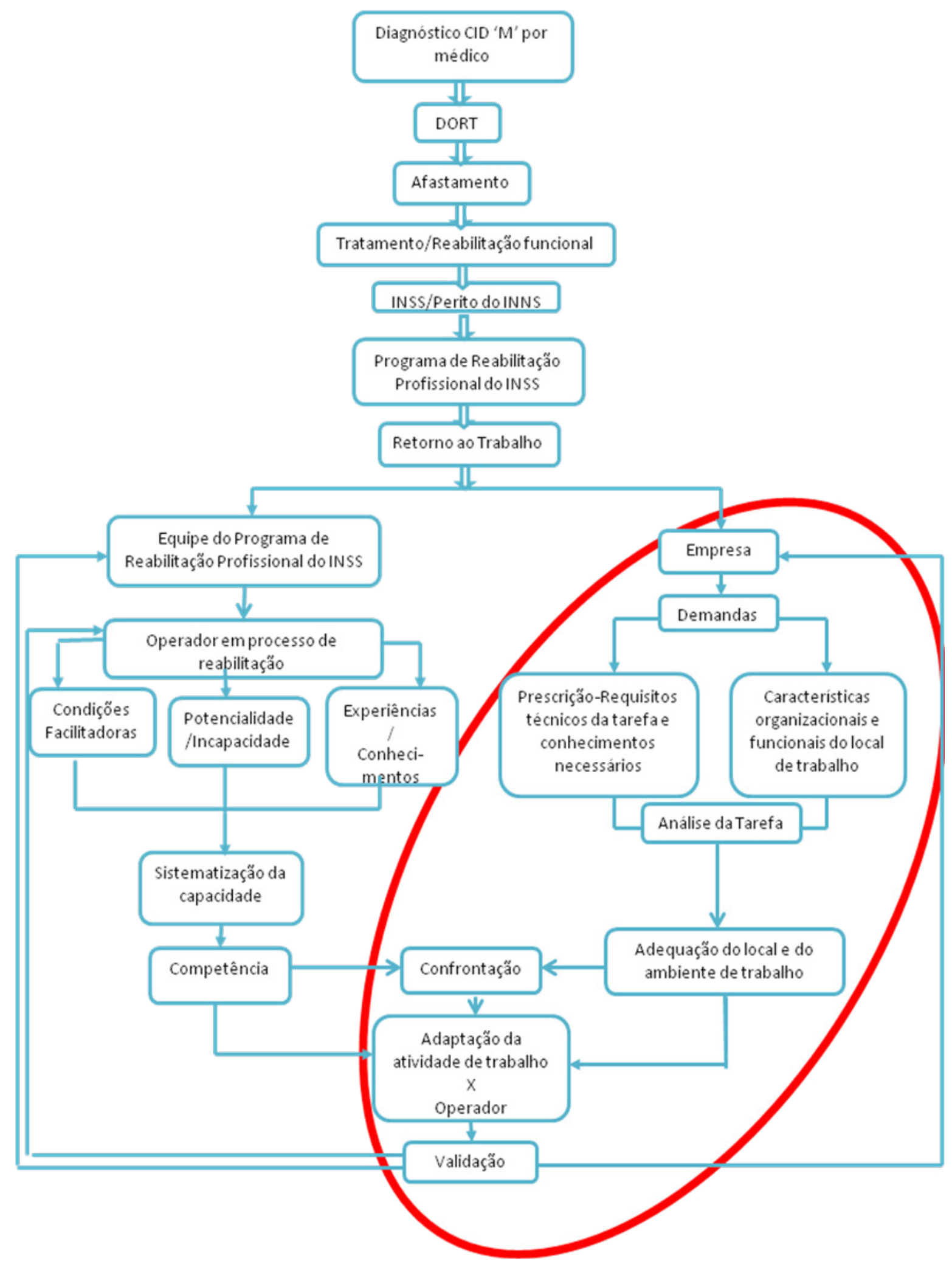

Fonte: adaptado de Silva e Camarotto ${ }^{17}$

Figura 1 - Fluxograma de inclusão da pessoa que retorna ao trabalho, após a identificação de LER/DORT 
A validação entre os três atores sociais é essencial para a análise da atividade de trabalho, visto que é um processo que tem seus alicerces construídos em conjunto e precisa de um acabamento que condiz à realidade do retorno.

A análise do posto de afastamento e posto de reinserção poderá demonstrar se houve ou não mudança significativa para o trabalhador no que diz respeito aos aspectos físicos (espaço, manuseio de carga e postura), nos aspectos organizacionais (repetitividade e conteúdo da atividade) e nos aspectos cognitivos (nível de atenção e vigilância, exigidos pela atividade e tomada de decisão).

A narrativa do trabalhador por meio de entrevista traz dados importantes quanto ao aspecto psicossocial do trabalho, ou seja, se está satisfeito com o novo posto; se contribuiu nesse processo; como se sente frente aos colegas de trabalho; como se sente frente ao novo posto de trabalho.

A entrevista é definida como uma conversa a dois, feita por iniciativa do entrevistador, destinada a fornecer informações pertinentes para um objeto de pesquisa. Sua utilização permite apresentar as experiências vividas por um indivíduo, um grupo ou uma organização e é capaz de verificar o conteúdo cotidiano, as opiniões e motivações dos participantes da história, protagonistas dos fatos sociais ${ }^{19}$.

\section{CONCLUSÃO}

A análise da atividade permite a compreensão dos aspectos intrínsecos das ações dos trabalhadores e os respectivos constrangimentos, o que permite adequar a demanda da empresa com as potencialidades dos trabalhadores.

Aoperacionalização do processo de retorno ao trabalho, no que diz respeito à preparação da empresa para a inclusão e percepção do trabalhador nesse processo, demonstra-se como necessidade do mundo do trabalho, em que se verifica que os atores sociais, trabalhador, empresa e Programa de Reabilitação Profissional do INSS, encontram-se pouco articulados.

No processo de retorno e inclusão no trabalho, é necessário que os técnicos do Programa de Reabilitação Profissional e da empresa, tenham conhecimento sobre o trabalho realmente realizado pelos trabalhadores, reconhecendo suas potencialidades e capacidades laborais. A metodologia da AE e uso da CIF permitem esta melhor compreensão dos postos de trabalho e potencialidades do trabalhador, de forma que a análise vá além dos aspectos físicos do trabalho, mas abranja os aspectos cognitivos e organizacionais, que são pauta de estudos aprofundados hoje na ergonomia.

Este estudo encontra-se na fase do desenvolvimento de campo em situações reais de trabalho para a validação da proposta, visando contribuir para futuro modelo de análise no retorno do trabalhador afastado por LER/DORT.

Autoria e Indicação de Responsabilidade: EC Silva trabalhou na concepção e na redação do artigo; JA Camarotto fez uma revisão crítica e com sugestões para a versão a ser publicada.

\section{REFERÊNCIAS}

1. Lancman S, Toldrá RC, Santos MC. Reabilitação profissional e saúde mental no trabalho. In: Glina DMR, Rocha LE. Saúde mental no trabalho da teoria à prática. São Paulo: Roca; 2010. p.98-112.

2. Maggi, B. Do agir organizacional - um posto de vista sobre o trabalho, o bem-estar, a aprendizagem. São Paulo: Edgard Blücher; 2006.

3. Brasil. Ministério da Previdência Social. Aeps Infologo - Base de dados históricos da Previdência Social, 2013. Brasília; 2013 [citado 28 mar. 2016]. Disponível em: http:// www3.dataprev.gov.br/scripts10/dardoweb.cgi.

4. Rossi EZ. Reabilitação e reinserção no trabalho de bancários portadores de LER/DORT: análise psicodinâmica
[Tese]. Brasília: Universidade de Brasília, Instituto de Psicologia; 2008. Disponível em: http://repositorio.unb.br/ bitstream/10482/1340/1/TESE_2008 ElisabethZRossi.pdf.

5. Brasil. Constituição Federal. Título VII. Da ordem social. Capítulo I. Art. 193 e 194. Diário Oficial da União, Brasília, 05 de outubro de 1988.

6. Maeno M, Takahashi MAC, Lima MAG. Reabilitação profissional como política de inclusão social. Acta Fisiátr. 2009;16(2):53-8. DOI: http://dx.doi.org/10.1590/S030376572013000100014 .

7. Takahashi MAC. Incapacidade e previdência social: trajetória de incapacitação de trabalhadores adoecidos por LER/DORT no contexto da reforma previdenciária brasileira da década de 
1990 [Tese]. Campinas: Universidade Estadual de Campinas; 2006. Disponível em: http://www.bibliotecadigital.unicamp. $\mathrm{br} /$ document $/$ ?code $=$ vtls000386685.

8. Guérin F, et al. Compreender o trabalho para transformá-lo: a prática da ergonomia. São Paulo: Edgar Blücher; 2001.

9. Lancman S, Daldon MT, Barros J, Gonçalves R, Jardim T. Processos de retorno e permanência no trabalho: elementos estruturantes para a construção de um modelo de ação. In: Simonelli AP, Rodrigues DS, organizadores. Saúde e trabalho em debate: velhas questões, novas perspectivas, Brasília: Ed. Paralelo 15; 2013. p.135-57.

10. Árnetz BB, Sjögren B, Rydéhn B, Meisel R. Early workplace intervention for employees with musculoskeletal-related absenteeism: a prospective controlled intervention study. J Occup Environ Med. 2003;45(5):499-506.

11. Meijer EM, Sluiter JK, Frings-Dresen MHW. Evaluation of effective return to work treatment programs for sicklisted patients with non-specific musculokeletal complaints: a systematic review. Int Arch Occup Environ Health. 2005;78:523-32. DOI: 10.1007/s00420-005-0622-x.

12. Toldrá RC, Daldon MTB, Santos MC, Lancman S. Facilitadores e barreiras para o retorno ao trabalho: a experiência de trabalhadores atendidos em um Centro de Referência em Saúde do Trabalhador - SP, Brasil. Rev Bras Saude Ocup (São Paulo). 2010;35(121):10-22. DOI: 10.1590/S0303-76572010000100003.

13. Saldanha JHS, Pereira APM, Neves RF, Lima MAG. Facilitadores e barreiras de retorno ao trabalho de trabalhadores acometidos por LER/DORT. Rev Bras Saude Ocup (São Paulo). 2013;38(127):122-38. DOI: http://dx.doi. org/10.1590/S0303-76572013000100014.

14. Simonelli AP, Camarotto JA, Bravo ES, Vilela RAG. Proposta de articulação entre abordagens metodológicas para melhoria do processo de reabilitação profissional. Rev Bras Saude Ocup (São Paulo). 2010;35(121):64-73. DOI: http:// dx.doi.org/10.1590/S0303-76572010000100008.

15. Wisner A. A metodologia na ergonomia: ontem e hoje. In: Wisner A. A inteligência no trabalho: textos selecionados de ergonomia. São Paulo: FUNDACENTRO; 1994. p.87-107.

16. Camarotto JA, Simonelli AP, Rodrigues DS. Ergonomia e trabalho. In: Simonelli AP, Rodrigues DS, organizadores. Saúde e trabalho em debate: velhas questões, novas perspectivas. Brasília: Ed. Paralelo 15; 2013. p.33-53.

17. Silva EC, Camarotto JA. Análise da atividade no processo de retorno ao trabalho do trabalhador afastado por DORT. In: Anais do $17^{\circ}$ Congresso Brasileiro de Ergonomia, 2014.

18. Simonelli AP. Contribuições da análise da atividade e do modelo social para a inclusão no trabalho de pessoas com deficiência [Tese]. São Carlos: Programa de Pós Graduação em Engenharia de Produção, Universidade Federal de São Carlos; 2009. Disponível em: http://www.dominiopublico. gov.br/pesquisa/DetalheObraForm.do?select_action $=\& \mathrm{co}_{-}$ obra $=149206$.

19. Minayo MCS. O desafio do conhecimento: pesquisa qualitativa em saúde. São Paulo: Hucitec-Abrasco; 1992.

Recebido em: 30.10 .15

Aceito em: 04.05.16 